\title{
12
}

\section{PROPERTIES OF GOLD NANOANTENNAS IN THE INFRARED}

\author{
F. Neubrech, J. Aizpurua, S. Karim, T. W. Cornelius, and A. Pucci
}

W

ith infrared spectroscopic microscopy using synchrotron light, resonant light scattering from single gold nanowires was investigated. The nanowires with diameters in the range of $100 \mathrm{~nm}$ were prepared by electrochemical deposition in polycarbonate etched ion-track membranes and transferred onto infrared-transparent substrates. For a few microns long nanowires, antenna-like plasmon resonances were observed in agreement with light scattering calculations. The resonances are dependent on wire length, wire shape, and on the dielectric surroundings of the nanowire. Electromagnetic far-field enhancement at resonance points to the ability of the nanowire to confine light on the nanoscale. This effect can be exploited for surface enhanced infrared absorption.

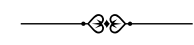

\subsection{Introduction}

Below the onset of interband transitions, the infrared (IR) optical properties of metals are determined by the collective oscillations of free 
charge carriers called plasmons. The Drude dielectric function

$$
\varepsilon(\omega)=\varepsilon_{\infty}-\frac{\omega_{\mathrm{p}}^{2}}{\omega\left(\omega+\mathrm{i} \omega_{\tau}\right)}
$$

is a good description of that behaviour. ${ }^{1-5}$ Effects from interband transitions on the background polarizability are included in $\varepsilon_{\infty}$. The Drude parameters $\omega_{\mathrm{p}}$ and $\omega_{\tau}$ describe the plasma frequency and the relaxation rate of the free charge carriers, respectively. With $\omega_{\mathrm{p}}$ much higher than IR frequencies and $\omega_{\tau} \ll \omega_{\mathrm{p}}$ the Drude dielectric function has a strong negative real part in the IR and corresponds to a considerably high polarizability. Above (circular) frequency $\omega=\omega_{\mathrm{p}} / \sqrt{\varepsilon_{\infty}}$ (the socalled plasma edge) the high metallic reflectivity drops down to values that depend on background polarizability and interband transitions. The high reflectivity at lower frequencies is related to the small penetration depth (skin depth) of the IR light into the metal. For $\omega_{\tau}$ in the mid IR (typical for iron or defect-rich noble metals) the skin depth in the $\mathrm{IR}^{6}$ is

$$
\delta=\frac{c}{\omega_{\mathrm{p}}} \sqrt{\frac{2 \omega_{\tau}}{\omega}}
$$

with $c$ as the velocity of light. Typical values for skin depths in the mid IR are 10 to $50 \mathrm{~nm}$ for metals. Therefore, metal films of a few $\mathrm{nm}$ in thickness are partially transparent in the IR. This behaviour extends to the visible range where (for sufficiently large $\omega_{p}$ ) in case of oblique incidence of p-polarized light the transmittance spectra of ultrathin metal films show the additional plasmon resonance around $\omega=\omega_{\mathrm{p}} / \sqrt{\varepsilon_{\infty}}$ due to the dispersion of surface plasmon polaritons. ${ }^{7}$ This resonance corresponds to collective charge carrier oscillations (plasmons) in perpendicular direction for which the film boundaries are effective. For metal particles the size and shape determine the boundary conditions for the plasmon oscillations. Vice versa, the optical resonances can be tuned via shape and size. The tunable optical properties of metal nanoparticles and their applications have been a topic of dramatically increasing interest over the last years ${ }^{8-11}$. In particular, this interest is related to the considerable electromagnetic field enhancement in the near-field of a metal nanoparticle at resonance. As an example, it can be used to control field amplification in surface enhanced Raman scattering (SERS) ${ }^{12,13}$. Extremely high near-field enhancement was calculated for the region of a 
slit between nanorods ${ }^{14}$. Related to this, electromagnetic resonances of nano-objects can be exploited to confine light to a volume of subwavelength dimensions ${ }^{15,16}$. Arrays of nanosized objects exhibit interesting photonic properties ${ }^{17}$ and composites of nanostructures are the focus of search for metamaterials ${ }^{18-20}$. Compared to antenna-like resonances in the visible range ${ }^{21}$, electromagnetic field enhancement by metal antennas is higher in the infrared because of the strong negative part of the metal dielectric function and the high aspect ratios that can be produced. The resonant modes of collective charge carrier motion that can be excited by electromagnetic radiation with wavelength much larger than the particle size are mainly determined by the material properties of the nanoparticles. ${ }^{22}$ Going from spherical particles to prolate spheroids, the shape dependence of the resonances becomes much stronger ${ }^{23,24}$ mainly for electric field along the longer particles axis. The plasmon resonance splits into branches with a low-energy mode, which can be detected in the mid-infrared for an adequate length of the nanoparticle ${ }^{25}$. The larger the aspect ratio (long axis $L /$ small axis $D$ ) of the particle the lower the frequency of this mode. When the wire length approaches the range of the exciting wavelength, the effects of retardation dominate the resonance condition and the limited speed of light leads to a direct dependence of the resonance frequency on the absolute size of the particle. This is well known from purely classical theory of scattering of electromagnetic waves by ideal metal objects ${ }^{26}$. Here antenna resonances occur if the length $L$ of an infinitely thin wire roughly matches with multiples of the wavelength $\lambda$, i.e.

$$
L=\frac{\lambda}{2 n} \cdot m \text {, }
$$

where $m$ is a natural number and $n$ is the refractive index of the surrounding medium. The fundamental mode of the resonance corresponds to $m=1$, higher dipole-like excitations correspond to odd $m$. If the diameter $D$ of the antennas is no longer negligible compared to the wavelength, the resonance frequency depends also on $D^{26}$. Further, the skin effect has to be considered if $D$ is in the range of the skin depth $\delta$. In that case, the skin effect has an impact on the dependence of the antenna resonance on the material properties ${ }^{27}$ and introduces a qualitative change of the resonance relation ${ }^{14}$. The change caused by the influence of $\delta$ on the resonance condition is opposite to the geometrical one caused by a non-negligible $D$. Through the ratio $\delta / D$ the Drude parameters influence the resonance curve of a nanoantenna. In particular, high defect density inside the metal particles would increase $\omega_{\tau}$ and thus 
the ratio $\delta / D$. In addition to the above mentioned effects, via the boundaries, the polarizability of the surrounding medium also affects the optical properties of metal nanoparticels ${ }^{28}$. In order to take into account the effect of a substrate (with the refractive index $\varepsilon_{\mathrm{s}}$ ) an effective medium completely embedding the wires is a good approximation. With its effective dielectric constant

$$
\varepsilon_{\text {eff }}=\frac{1}{2}\left(1+\varepsilon_{\mathrm{s}}\right)
$$

and $n=n_{\text {eff }}=\sqrt{\varepsilon_{\text {eff }}}$ the influence of the substrate on optical spectra can be described well ${ }^{22,29}$.

\subsection{Measurement of Antenna Resonances in the Infrared}

The clearest experimental result is expected for individual gold wires with a cylindrical shape, a low defect density, and a clean surface. Gold is preferred because of its stability under ambient conditions. Gold wires with diameters between 60 and $200 \mathrm{~nm}$ were prepared, for example, by electrochemical deposition in etched ion-track membranes ${ }^{30,31}$. Fig. 12.1 shows a scanning electron microscopy (SEM) image of a gold nanowire (with $D=100 \mathrm{~nm}$ ), displaying its smooth contour and regular shape. Transmission electron microscopy studies proved that gold nanowires deposited under the conditions mentioned above are polycrystalline ${ }^{30}$. After formation of wires with a controlled length, the polymer membrane was dissolved in dichloromethane in order to achieve clean nanowires.

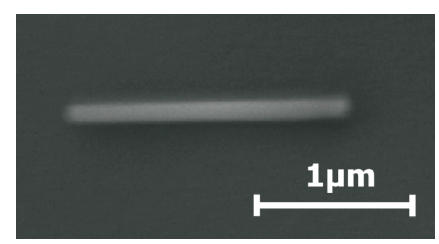

Fig. 12. 1 SEM image of a gold wire with $100 \mathrm{~nm}$ in diameter, see text.

For IR spectroscopy these wires were transferred to different IR transparent substrates $\left(\mathrm{ZnS}, \mathrm{KBr}\right.$, and $\left.\mathrm{CaF}_{2}\right)$, with different refractive indices $n_{\mathrm{s}}\left(n_{\mathrm{ZnS}}=4.84\right.$ at $10 \mu \mathrm{m}, n_{\mathrm{KBr}}=1.53$, and $n_{\mathrm{CaF} 2}=1.4$ at $\left.5 \mu \mathrm{m}\right)$. During the sample preparation process, the several microns long wires broke into pieces with lengths between several hundred $\mathrm{nm}$ and few $\mu \mathrm{m}$, and with tip ends determined by the grain-boundary structure of the wires. 
IR spectroscopic microscopy of single gold nanowires, produced as explained above, was performed at the IR beamline of the synchrotron light source ANKA (Angströmquelle Karlsruhe) at the Forschungszentrum Karlsruhe. Previous to wire-array studies, the individual wires were randomly placed on the IR transparent substrate and, therefore, their position needed to be located. At the beamline this and the length determination were done with optical microscopy with light (which gives an error of up to $8 \%$ ). Then the IR-beam spot with diameter of about $8 \mu \mathrm{m}$ was centered on the selected nanowire and IRtransmittance spectra (sample spectra) were recorded. Subsequently, in order to eliminate environmental effects like beam profile and substrate inhomogeneities, reference spectra were taken at least $10 \mu \mathrm{m}$ away from any nanowire. The spectroscopic measurements were done with a Fourier-transform infrared (FT-IR) spectrometer (Bruker IFS $66 \mathrm{v} / \mathrm{S}$ ) and a LN2-cooled mercury-cadmium telluride (MCT) detector, which collects light normally transmitted through the sample area. A small fraction of the light scattered away from the normal direction was also detected due to the collection lens (Schwarzschild objective, numerical aperture: 0.52), but its intensity was negligibly small. For both sample and reference measurements IR spectra were recorded by acquisition of at least 10 scans in the spectral range from 600 to $7000 \mathrm{~cm}-1$ with a resolution of at least $16 \mathrm{~cm}-1$. For further analysis of the transmitted light, a polarizer was inserted in the optical path before the sample.

For nanowires with a length of a few microns significant fundamental antenna like plasmon resonances appear in the relative transmittance spectra (ratio of the sample and reference spectra, see Fig. 12.2). Apart from the fundamental resonance, much weaker structures of resonant extinction can be found at higher wavenumbers. Usually they are attributed to higher order resonances. The fundamental resonance was observed only for electrical field parallel to the long axis of the wire (parallel polarization). For polarization perpendicular to the long wire axis (perpendicular polarization), the IR signal was below the noise level. That behaviour proves the dipole character of the excitation attributed to $m=1$. 


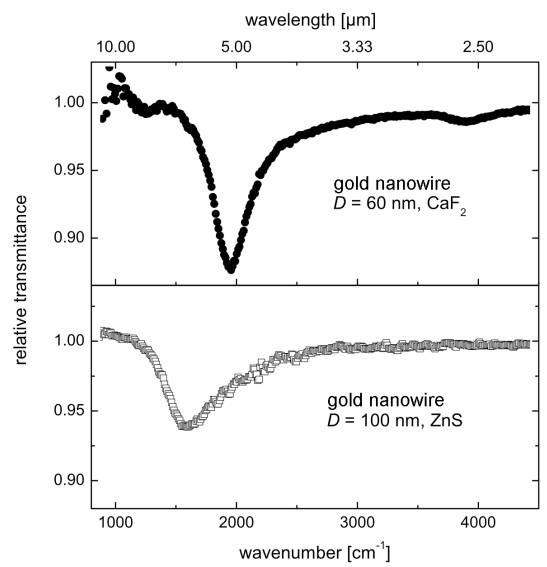

Fig. 12.2 IR transmittance spectra of two individual gold nanowires on different substrates (as indicated) normalized to the transmittance of the bare substrate. At the lower wave numbers the $\mathrm{CaF}_{2}$ substrate arrives at the multi-phonon edge, which lowers the photon intensity at the detector, which gives rise to noise in the spectrum. The electric field was parallel to the wire.

From the measured relative transmittance spectra the ratio of the extinction cross section $\sigma_{\text {ext }}$ of a single gold nanowire to its geometric cross section $\sigma_{\text {geo }}$ can be estimated. As we already reported in reference 29 , we use the relation

$$
\frac{\sigma_{\text {ext }}}{\sigma_{\text {geo }}}=A_{0}\left(1-T_{\text {rel }}\right) \cdot\left(n_{\mathrm{s}}+1\right) \cdot \frac{1}{(2 L D)},
$$

where the substrate screening is taken into account in analogy to the normal transmittance change by a thin film on a substrate ${ }^{32}$ in comparison to a free-standing film ${ }^{33}$. Inserting $A_{0}$ as the spot size, $\left(1-T_{\text {rel }}\right)$ the transmittance change in the spectrum, $n_{\mathrm{s}}$ for the relevant substrate, $L$ from dark-field microscopy in the visible spectral range, and $D$ (known from wire-growth process) lead to $\sigma_{\text {ext }} / \sigma_{\text {geo }}$ as shown for one example in Fig. 12.3. Any value $\sigma_{\text {ext }} / \sigma_{\text {geo }}>1$ means an extinction of intensity above pure shadowing, which indicates a local field enhancement in the vicinity of the nanowire. From the cross section ratio $\sigma_{\text {ext }} / \sigma_{\text {geo }}$ the spatially averaged field enhancement in the far-field $\left(\sqrt{\sigma_{\text {ext }} / \sigma_{\text {geo }}}\right)$ can be calculated. 


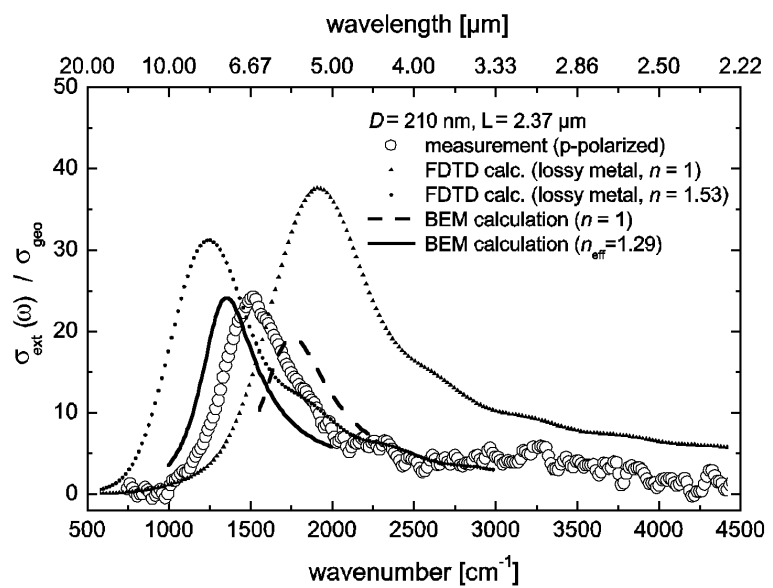

Fig. 12.3 Extinction cross section (normalized to geometric cross section): experimental curve for a gold nanowire on $\mathrm{KBr}$ compared to calculations for different effective refractive indices around the nanowire, see text.

The experimental spectrum that is shown in Fig. 12.3 represents the best of our measurements and reveals far-field enhancement by a factor of about 5 at the resonance maximum. The other curves were derived with two different theoretical methods. In both the methods the gold nanowire is modelled as a rod with hemispherical tip ends completely embedded in a medium (background). The far-field observation point in both calculations is in the plane defined by the rod axis and the light propagation axis. Those resonance curves marked by "FDTD calc." were modelled with the software CST Microwave studio ${ }^{34}$ for antenna problems and were based on a finite difference time domain (FDTD) algorithm $^{35}$. In this computational approach, Maxwell's time dependent differential equations are solved over a three-dimensional grid. In our calculations one single gold nanowire was illuminated by a plane wave polarized along the long wire axis, and the antenna radiated into free space. The curves marked with "BEM calculations" were calculated with the exact boundary element method (BEM) including retardation ${ }^{14,36}$. Maxwell's equations for inhomogeneous media with sharp boundaries were solved in terms of charges and currents distributed on the surfaces and interfaces. Boundary conditions are imposed via surface integrals along the boundaries between different media. Each region is characterized by a local dielectric function. The external fields interact self-consistently with the induced boundary charges and currents, which are determined by discretizing the surface integrals and solving the 
appropriate matrix equations. In this approach, the scattered field due to an incident external field is calculated directly.

In the FDTD calculations, we described the metal of the gold nanowires as a lossy one. The "lossy metal" model neglects the imaginary part of the conductivity. Changing the background material from air to a dielectric one $(\mathrm{KBr})$ a shift of the resonance maximum towards smaller wavenumbers is observed (dotted line). Obviously, neither the calculation based on vacuum as background nor the curve with background $\mathrm{KBr}$ describes the measured data. The resonance curves calculated with the boundary element method (BEM) are in better agreement with the experiment. Using an effective refractive index $n_{\text {eff }}=$ 1.29 the best agreement, in strength and shape, of the resonance curve with the experimental data is obtained. Only the position of the extinction maximum differs slightly, which can be related to the error in the real length of the wire.

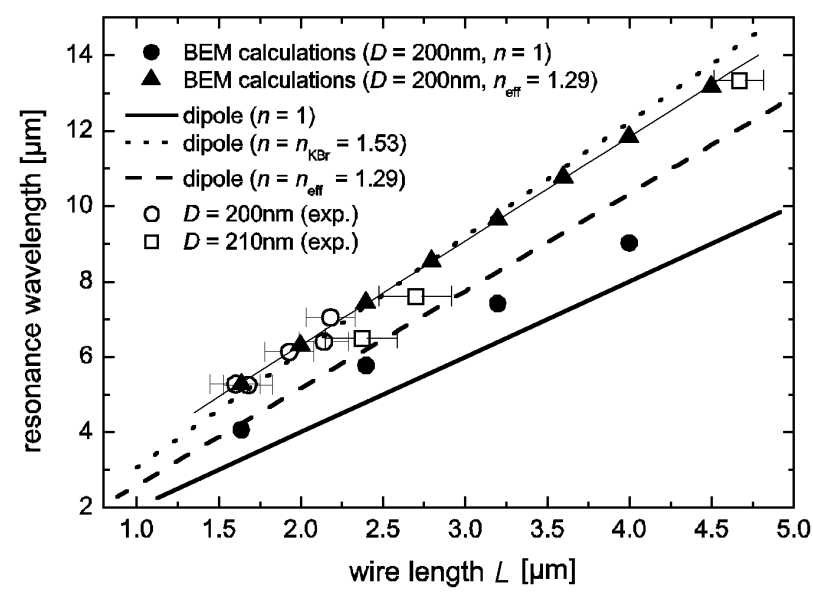

Fig. 12.4 Resonance wavelength versus wire length $L$ for gold nanowires with comparable diameter on $\mathrm{KBr}$. The figure shows experimental data (open symbols), idealantenna model calculations for wires embedded in different surroundings (lines), and detailed BEM calculations. The lines corresponding to Eq. $3(m=1)$ are shown for comparison.

Looking at Fig. 12.4, where we display the resonance wavelengths, from the measured spectra for nanowires with comparable diameters on $\mathrm{KBr}$, systematic deviation from simple antenna theory (black line) is obvious. When using the value of the refractive index of $\mathrm{KBr}$ in $\mathrm{Eq} .3$ the calculated curve (dotted line) seems to fit our measured data. But this in conflict with the fact that the wires are not embedded in this material. On 
the other hand, BEM calculations for wires embedded in an effective medium with refractive index $n_{\text {eff }}=\sqrt{\varepsilon_{\text {eff }}}$ yield good agreement with the measured data. Also the effect of a cover medium is well described by the respective $\varepsilon_{\text {eff }}=\left(\varepsilon_{\mathrm{m}}+\varepsilon_{\mathrm{s}}\right) / 2$ (for surrounding medium with dielectric constant $\left.\varepsilon_{\mathrm{m}}\right)$ as it is shown by the coverage of individual gold nanowires on different substrates $(\mathrm{KBr}, \mathrm{ZnS})$ with paraffin wax. Due to the paraffin coverage the resonance is shifted to lower frequencies ${ }^{29}$.

The theoretical ratio is $n_{\text {eff }}^{\text {air }} / n_{\text {eff }}^{\text {parffin }}=0.875$ on $\mathrm{KBr}$ and 0.923 on $\mathrm{ZnS}$ (with $\varepsilon_{\mathrm{m}}=2.02$ for paraffin), respectively. These ratios are in reasonable accord with the results obtained from the spectral shift of the resonance frequencies before and after evaporating paraffin.

\subsection{Surface Enhanced Infrared Absorption (SEIRA)}

Field enhancement in the IR can be exploited for surface enhanced infrared absorption (SEIRA) $)^{37,38}$ of molecules. In case of sufficiently strong near-field enhancement, SEIRA would allow the detection of vibrational fingerprints of single molecules on nanoantennas, for example, by studying light scattering with aperture-less scanning nearfield microscopy ${ }^{39}$.

Until now SEIRA is less well-known than SERS since its signal enhancement was measured to be only three orders of magnitudes ${ }^{37,38}$. This is much less than the SERS enhancement which is many more orders of magnitude. This difference is related to the different influence of local electromagnetic field enhancement. In SEIRA the squared nearfield enhancement is relevant, whereas in SERS, which is a scattering process, two kinds of squared field enhancements are multiplied (one for the incident field and the other one for the scattered field). ${ }^{40}$ Usually, the electromagnetic field enhancement is obtained with metal nanoparticles or nanostructured metal films. Their plasmonic resonances give rise to near-field enhancement as the main effect in SEIRA and SERS ${ }^{41}$. Other contributions to the observed enhancement of vibration signals are less important (concerning the contribution to enhancement) and less well understood. They are related to chemical interactions and non-adiabatic effects and are relevant for the first adsorbate layer only. ${ }^{42}$

The near-field enhancement is strongest in the proximity of sharp edges or tips. For SERS this tip effect was already studied by several groups. ${ }^{12,43}$ For nanoantennas in the IR such tip effect should be much more like a lightning rod effect. This would means huge near-field enhancement at the tip ends of an antenna at resonance, much larger than 
three orders of magnitude as measured as the spatially averaged enhancement for adsorbates on metal-island films compared to the same adsorbate layer without metal islands.

\section{Acknowledgement}

F. N. and A. P. gratefully acknowledge technical support by M. Süpfle, helpful discussions with A. Otto, B. Gasharova, and Y.-L. Mathis. J.A. acknowledges software access from F.J. García de Abajo, discussions with G.W. Bryant, and financial support from Gipuzkoa Foru Aldundia (Gipuzkoa Fellows program) and the Government of the Basque Country (NANOTRON project).

\section{References}

1. G. Fahsold, A. Bartel, O. Krauth, N. Magg and A. Pucci, IR-optical properties of ultrathin Fe films on $\mathrm{MgO}(001)$ beyond the percolation threshold, Phys. Rev B 61, 14108 (2000)

2. G. Fahsold, M. Sinther, A. Priebe, S. Diez and A. Pucci, Adsorbateinduced changes in the broadband infrared transmission of ultrathin metal films, Phys. Rev. B 65, 235408 (2002)

3. G. Fahsold and A. Pucci, Non-contact measurement of thin-film conductivity by ir spectroscopy, Advances in Solid State Physics (ed. by B. Kramer, Springer press) 43, 833 (2003)

4. A. Pucci, F. Kost, G. Fahsold and M. Jalochowski, Infrared spectroscopy of $\mathrm{Pb}$ layers growth on Si (111), Phys. Rev B 74, 125428 (2006)

5. F. Meng and A. Pucci, Growth of silver on $\mathrm{MgO}(001)$ and infrared optical properties, phys. stat. sol. (a) in press (2007)

6. F. Abeles, Optical properties of metals, in Optical properties of solids, ed. by F. Abeles (North-Holland Publishing, Amsterdam, 1972), pp. 93 
7. Y. Borensztein, M. Roy and R. Alameh, Threshold and Linear Dispersion of the plasma resonance in Thin Ag Films, Europhysics Lett. 31, 311 (1995)

8. S. Link and M. A. El-Sayed M A 1999, Spectral properties and relaxation dynamics of surface plasmon electronic oscillations in gold and silver nano-dots and nano-rods, J. Phys. Chem. B 103, 8410 (1999)

9. N. Félidj, J. Aubard, G. Lévi, J. R. Krenn, M. Salerno, G. Schider, B. Lambrecht, A. Leitner and F.R. Aussenegg, Controlling the optical response of regular arrays of gold particles for surface-enhanced Raman scattering, Phys. Rev. B 65, 075419 (2002)

10. G. Schider, J. R. Krenn, A. Hohenau, H. Ditlbacher, A. Leitner, F. R. Aussenegg, W. L. Schaich, I. Puscasu, B. Monacelli and G. Boreman, Plasmon dispersion relation of $\mathrm{Au}$ and $\mathrm{Ag}$ nanowires, Phys. Rev. B 68, 155427 (2003)

11. C. Soennichsen, T. Franzl, T. Wilk, G. von Plessen, J. Feldmann, O. Wilson and P. Mulvaney, Drastic reduction of plasmon damping in gold nanorods, Phys. Rev. Lett 88, 077402 (2002)

12. J. Grand, M. Lamy de la Chapelle, J. L. Bijeon, P. M. Adam, A. Vial and P. Royer, Role of localized surface plasmons in surfaceenhanced Raman scattering of shape-controlled metallic particles in regular arrays, Phys. Rev. B 72, 033407 (2005)

13. H. Xu, E. J. Bjerneld, M. Kaell and L. Boerjesson, Spectroscopy of single hemoglobin molecules by surface enhanced Raman scattering, Phys. Rev. Lett. 834357 (1999)

14. J. Aizpurua, G. W. Bryant, L. J. Richter, F. J. García de Abajo, B. K. Kelley and T. Mallouk, Optical properties of coupled metallic nanorods for field-enhanced spectroscopy, Phys. Rev. B 71, 235420 (2005)

15. E. Cubukcu, E. A. Kort, K. B. Crozier and F. Capasso, Plasmonic laser antenna, Appl. Phys. Lett. 89, 093120 (2006) 
16. A. Maier, P. G. Kik and H. A. Atwater, Observation of coupled plasmon-polariton modes in Au nanoparticle chain waveguides of different lengths: Estimation of waveguide loss, Appl. Phys. Lett. 81, 1714 (2002)

17. M. Naruse, T. Kawazoe, S. Sangu, K. Kobayashi, and M. Ohtsu, Optical interconnects based on optical far- and near-field interactions for high-density data broadcasting, Opt. Express 14, 306 (2006)

18. R. Moussa, S. Foteinopoulou, L. Zhang, G. Tuttle, K. Guven, E. Ozbay and C. M. Soukoulis, Negative refraction and superlens behavior in a two-dimensional photonic crystal, Phys. Rev. B 71, 085106 (2005)

19. J. B. Pendry, Negative Refraction Makes a Perfect Lens, Phys. Rev. Lett. 85, 3966 (2000)

20. V. A. Podolskiy, A. K. Sarychev, E. E. Narimanov and V. M. Shalaev, Resonant light interaction with plasmonic nanowire systems, J. Opt. A: Pure Appl. Opt. 7, 32 (2005)

21. J. P. Schuck, D. P. Fromm, A. Sundaramurthy, G. S. Kino and W. E. Moerner, Improving the Mismatch between Light and Nanoscale Objects with Gold Bowtie Nanoantennas, Phys. Rev. Lett. 94, 017402 (2005)

22. for example: M. M. Dvoynenko, A. V. Goncharenko, V. R. Romaniuk V R and E. F. Venger, ) Effects of dimension on optical transmittance of semicontinuous gold, Physica B 29988 (2001)

23. J. Grand, P. M. Adam, A. S. Grimault, A. Vial, M. Lamy de la Chapelle, J. L. Bijeon, S. Kostcheev and P. Royer, Optical Extinction Spectroscopy of Oblate, Prolate, and Ellipsoid Shaped Gold Nanoparticles: Experiments and Theory, Plasmonics 1, 135 (2006)

24. A. Bouhelier, R. Bachelot, G. Lerondel, S. Kostcheev, P. Royer and G. P. Wiederrecht, Surface Plasmon Characteristics of Tunable Photoluminescence in Single Gold Nanorod, Phys Rev. Lett 95, 267405 (2005) 
25. K. B. Crozier, A. Sundaramurthy, G. S. Kino and C. F. Quate, Optical antennas: Resonators for local field enhancement, J. Appl. Phys. 9, 4632 (2003)

26. T. G. Ruck, Radar Cross Section Handbook (Plenum Press, New York, 1970)

27. R. B. Dingle, The Electrical Conductivity of Thin Wires, Proceedings of the Royal Society of London, Series A, Mathematical and Physical Sciences 2011067 (1950)

28. J. Mock, D. R. Smith and S. Schultz, Local refractive index dependence of plasmon resonance spectra from individual nanoparticles, Nanoletters 3, 485 (2003)

29. F. Neubrech, T. Kolb, R. Lovrincic, G. Fahsold, J. Aizpura, M. E. Toimil-Molares, S. Karim, T. W. Cornelius, R. Neumann and A. Pucci, Resonances of individual metal nanowires in the infrared, Appl. Phys. Lett. 89, 253104 (2006)

30. J. Liu, J. L. Duan, M. E. Toimil-Molares, S. Karim, T. W. Cornelius, D. Dobrev, H. J. Yao, Y. M. Sun, M. D. Hou, D. Mo, Z. G. Wang and R. Neumann, Electrochemical fabrication of single crystalline and polycrystalline Au nanowires: the influence of deposition parameters, Nanotechnology 17, 1922 (2006)

31. S. Karim, M. E. Toimil-Molares, F. Maurer, G. Miehe, W. Ensinger W, J. Liu, T. W. Cornelius and R. Neumann, Synthesis of gold nanowires with controlled crystallographic characteristics, Appl. Phys. A 84, 403 (2006)

32. U. Teschner and K. Huebner, IR-spectroscopic data of thin insulating films on semiconductors. New methods of interpretation and analysis. phys. stat. sol. (b) 159, 917 (1990)

33. D. W. Berreman, Infrared Absorption at Longitudinal Optic Frequency in Cubic Crystal, Phys. Rev. 1302193 (1963)

34. CST Computer Simulation Technology, Darmstadt, Germany 
35. K. S. Yee, Numerical solution of boundary value problems involving Maxwell's equations in isotropic media, IEEE Trans. Antennas Propag. 14, 302 (1966)

36. F. J. García de Abajo and A. Howie, Relativistic Electron Energy Loss and Electron-Induced Photon Emission in Inhomogeneous Dielectrics, Phys. Rev. Lett. 80, 5180 (1998) and Retarded field calculation of electron energy loss in inhomogeneous dielectrics, Phys. Rev. B 75, 115418 (2002)

37. R. F. Aroca, D. J. Ross and C. Domingo, Surface-Enhanced Infrared Spectroscopy, Applied Spectroscopy 58, 324A (2004)

38. D. Enders and A. Pucci, Surface enhanced infrared absorption of octadecanethiol on wet chemically prepared Au nanoparticle films, Appl. Phys. Lett. 88, 184104 (2006)

39. A. Cvitkovic, N. Ocelic, J. Aizpurua, R. Guckenberger and R. Hillenbrand, Infrared Imaging of Single Nanoparticles via Strong Field Enhancement in a Scanning Nanogap, Phys. Rev. Lett. 97, 060801 (2006)

40. A.W. Wokaun in: H. Ehrenreich, F. Seitz, D. Turnbull (Eds.), Surface enhanced Electromagnetic Processes, Solid State Phys, vol. 38, (Academic Press, New York, 1984), p 223

41. A. Otto, I. Mrozek, H. Grabhorn, and W. Akemann, Surface enhanced Raman scattering, J. Phys.: Condens. Matter 4, 1143 (1992)

42. A. Otto, The chemical (electronic) contribution to surface-enhanced Raman scattering, J. Raman spectroscopy 36, 497 (2005)

43. N. Calander and M. Willander, Theory of surface-plasmon resonance optical field enhancement at prolate spheroids, J. Appl. Phys. 92, 4878 (2002) 